\title{
EDITORIAL
}

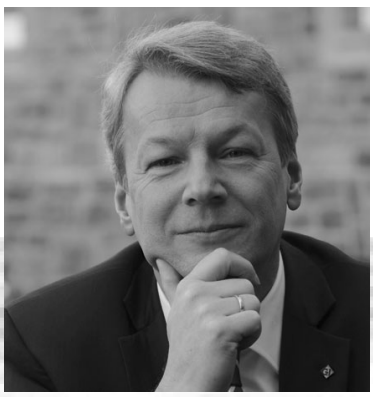

Peter Liggesmeyer

Foto: Cornelia Winter, GI

(Gesellschaft für Informatik)

\section{Deutschlands digitale Köpfe}

Als Präsident der Gesellschaft für Informatik e. V. (GI) freue ich mich aus mehreren Gründen über das 2014 seitens des BMBF initiierten Wissenschaftsjahr mit dem Thema "Die digitale Gesellschaft". Zum Einen ermöglicht dieses Thema in besonderer Weise die Herausstellung der überragenden Bedeutung der Informatik für alle Lebensbereiche in Beruf und Freizeit, von der IT bis zur Industrie, in der Medizin und Mobilität, in Politik und Gesellschaft sowie in Kunst, Kultur und Medien, um nur einige Beispiele zu nennen. Zum anderen hat die Gesellschaft für Informatik gemeinsam mit dem Bundesministerium für Bildung und Forschung im Rahmen des Wissenschaftsjahres das Projekt "Deutschlands digitale Köpfe" durchgeführt. Gegenstand war die Identifikation von Deutschlands digitalen Denkern und Machern mit den Zielen, Themen plastisch darzustellen und auch Empfehlungen in den einzelnen Handlungsfeldern zu erarbeiten.

Am 21. Mai 2014 hat sich eine hochkarätig und interdisziplinär besetzte Jury getroffen und Deutschlands „digitale Köpfe" gesucht und gefunden.

Siehe hierzu auch www.gi.de/digitale-koepfe.

Herausgekommen ist eine gelungene Mischung von 39 Frauen und Männern, die mit ihren inspirierenden Projekten, Ideen und Visionen Deutschlands digitale Exzellenz repräsentieren und die Zukunft der digitalen Gesellschaft maßgeblich vorantreiben. Die offizielle Kür der "digitalen Köpfe" fand auf der Gl-Tagung "die Zukunft der digitalen Gesellschaft" am 15. September 2014 in Berlin statt. Die dort erarbeiteten Handlungsempfehlungen sind der Bundesministerin Fr. Prof. Dr. Johanna Wanka am 2. Dezember 2014 übergeben worden. Ein weiterer Austausch unserer „Köpfe" mit dem BMBF und der GI steht zudem für 2015 auf der Agenda.

An dieser Stelle möchte ich mich beim BMBF für das schöne Wissenschaftsjahr, die gemeinsamen Aktivitäten und insbesondere auch für die Bereitschaft, die Schirmherrschaft über die Gl-Jahrestagung 2014 zu übernehmen, bedanken. Ich würde mich über weitere gemeinsame Schritte freuen.

Hier liegt nun ein Themenheft des Informatik Spektrums zu „Deutschlands digitalen Köpfen" vor. Fünf „Digitale Köpfe" diskutieren in jeweils einem Hauptbeitrag ihre jeweiligen Themen. Die Spanne reicht von verteilten intelligenten Systemen, über Big bzw. Smart Data, zukünftige Energiesysteme, digitalisierte Medizin bis hin zur Sozioinformatik. Diese Themenvielfalt, die allein von fünf der 39 "Köpfe" aufgespannt wird, belegt die Breite der Themen, die heute mit Informatik verbunden sind.

Zu dem finden Sie in dieser Ausgabe:

Manfred Nagl und Otto Spaniol - zwei Informatik-,,Urgesteine" diskutieren darüber, ob Informatik zum Allgemeingut und bedeutungslos wird oder eben nicht.

Und Kirstin Kohler vergleicht ihre Auslandserfahrungen im Silicon Valley mit der Situation in Deutschland: Nerds als Helden. Würden doch nur mehr Abiturientinnen und Abiturienten Informatik-Helden werden wollen! Gebrauchen könnten wir sie auf jeden Fall in den vielfältigen interessanten Anwendungsfeldern der Informatik.

Aber das ist anderes Thema, an dem wir bei der Gl auch intensiv arbeiten - die Informatik-Bildung.

Bleibt festzustellen: Wir können auf ein erfolgreiches Wissenschaftsjahr zurückschauen; wir haben "Digitale Köpfe"; wir haben eine digitale Agenda, und die Gl ist auf dem Weg in "die Mitte der Gesellschaft". Ich wünsche Ihnen viel Spaß bei der Lektüre dieses Hefts

$\mathrm{Ihr}$ 